Chapter 9

\title{
Neuroscience-Based Rehabilitation for Stroke Patients
}

\author{
Takayuki Kodama and Hideki Nakano
}

Additional information is available at the end of the chapter

http://dx.doi.org/10.5772/67440

\begin{abstract}
Hitherto, physical therapy for rehabilitating patients with cerebral dysfunction has focused on acquiring and improving compensatory strategies by using the remaining functions; it has been presumed that once neural functions have been lost, they cannot be restored. However, neuroscience-based animal research and neuroimaging research since the 1980s have demonstrated that recovery arises from plastic changes in the central nervous system and reconstruction of neural networks; this research is ushering in a new age of neuroscience-based rehabilitation as a treatment for cerebral dysfunction (such as stroke). In this paper, in regard to mental practices using motor imagery and kinaesthetic illusion, we summarize basic discoveries and theories relating to motor function therapy based on neuroscientific theory; in particular, we outline a novel rehabilitation method using kinaesthetic illusion induced by vibrational stimulus, which the authors are currently attempting in stroke patients.
\end{abstract}

Keywords: stroke, kinaesthetic illusion, motor imagery, neuroscience-based rehabilitation

\section{Introduction}

Conventional physical therapy (PT) for the rehabilitation of patients with brain dysfunction focuses on the acquisition of function through alternative means by using and improving the patients' existing functions, and it is based on the assumption that once a neutral function is lost, it can never be recovered [1]. However, animal neuroscience studies [2-4] that were conducted after the 1980s and neuroimaging studies [5, 6] have shown that recovery can occur as a result of plastic changes in the nervous system or reorganization of the neural network, and rehabilitation (neuroscience-based rehabilitation, NBR) after cerebral dysfunction (e.g. stroke) has reached a new era in treatment. These observations suggest that the plasticity that is observed in patients is related to the characteristic that the more the patient receives therapy 
in specific parts of their body, the more that the brain areas that control these parts will be functionally as well as anatomically extended.

Functional recovery originally referred to a patient's recovery from limitations in their behavior, movements, and/or activity [7]. Therefore, the purpose of NBR is not only to induce the reorganization of brain functions through neural plasticity mechanisms but also recover comprehensive bodily motor functions and brain functions for autonomous and active social behavior. What type of treatment strategy is required so that patients feel positively engaged by it, gradually understand its effects, and work toward a goal? Previous studies have revealed important factors in the effects of NBR treatment, such as the amount of therapy [8,9], rehabilitation implementation environment [10], and performance of neurocognitive rehabilitation [11] through mental practice techniques, such as motor imagery (MI) [12]. Among these factors, treatments involving MI are strongly recommended because MI contributes to the reorganization of neural functions. MI, which is an approach that is based on neuroscientific data and the motor learning theory, is defined as the capacity to internally mimic physical movements without any associated motor output [13]. The cognitive process that occurs during the imagination of movements involves various components, such as mutual understandings between oneself and others (environment), observations of movements, mental manipulations of objects, and psychological time and movement planning. Instead of repeating simple physical movements to receive feedback on outcome in the actual therapy, the practice of voluntary and skill-requiring movements that are geared toward task completion induces the functional recovery [14]. Thus, an important element of the patients' engagement in the therapy is that it occurs in an active and top-down fashion through the use of MI. However, because MI has a task-specific nature, cognitive functions and memories of motor experiences that equip the patients to perform the task are required. Patients with neurofunctional states that make motor execution (ME) difficulty may suffer not only from impairments in motor-related brain areas but also from modifications in their intracerebral body representations (e.g. somatoparaphrenia) $[15,16]$. In such cases, the exploitation of kinaesthetic illusions [17-20], which can be induced in the brain by extraneous stimuli, such as vibratory stimulations, becomes important for inputting appropriate motorsensory information into the brain in a passive and bottom-up fashion. Therefore, the implementation of a mental practice to determine the criteria for adequate treatment according to the states of the patient's cognitive functions and motor functions is important in order to select and implement the best therapy. Thus, this paper summarizes the basic understanding and theories of mental practices that use MI or kinaesthetic illusion and discusses, in particular, research results concerning kinaesthetic illusions that are induced by vibratory stimulations, which we are currently attempting on stroke patients.

\section{What is neuroscience-based rehabilitation?}

NBR involves a series of processes that are selected for the intervention according to the current brain function theories that have been revealed by neuroscience and other similar studies and verification of its outcomes. For example, the selection of a NBR strategy for a stroke patient requires a combination of deep clinical reasoning, the experience of the 
therapist, and a vast understanding of the evidence obtained by studies from wide-ranging academic fields on the factors that support recovery mechanisms and produce particular outcomes. First, the neural basis of brain cell reorganization will be presented.

\subsection{Neural basis of brain cell reorganization}

The current understanding of neural reorganization after dysfunction is not that the neurons themselves recover after their axons are damaged but rather that damaged functional networks recover due to several processes that induce the recovery of motor and cognitive functions. Cajal [1], who was a proponent of neuron theory, stated that the central nervous system (brain and spinal cord) of adult mammals would not recover once it is damaged. However, studies that have been conducted since the 1980s and that have shown that alterations in the peripheral nervous system, such as denervation and amputation, change somatic sensations and the representations of body parts while they are in motion have revealed that the brain has plasticity. In 1998, Eriksson et al. [21] reported the new formation of neurons in the central nervous system of human beings. These findings raised the question of whether the plastic changes and functional reorganization that occur in subjects with cranial nerve disorders originate from an ischemic state, such as a cerebrovascular disturbance. The underlying mechanisms of the plasticity that occurs after a cortical deficit are thought to involve (i) the redundancy of neuronal connections in the central nervous system, (ii) morphological changes in the neurons, and (iii) changes in synaptic information transmission [22]. If neurons are damaged, astrocytes begin to divide due to the activity of microglia. These glial cells then reinforce the areas that have been damaged by brain lesions and release neurotrophic factors, such as nerve growth factor, to promote neuronal sprouting (it takes around two weeks for synapses to grow after nerve damage [23]). The sprouted neurons are then connected to an existing neural network, which forms a new network. In other words, if neurons are damaged, new neurons begin to reorganize themselves in order to compensate for it. Adequate NBR stimulates the neural network with the neurofunction that is most similar to the predamaged functional state of the neural network, even though the new network is not located in the damaged region. If strong inputs enter the network multiple times, the synaptic connections will be reinforced. However, plasticity will not be induced in synapses with little information (input specificity), and the synapses will be excluded from the network formation [24, 25].

These findings have been confirmed by several famous studies. Nudo et al. [8] caused artificial cerebral infarcts in monkeys in the region of the primary motor cortex (M1) that corresponds to fingers and then forced the monkeys to use fingers with motor deficits. Thus, they reported that the brain region that previously controlled the shoulders and elbows prior to the therapy then controlled the fingers and more distal body parts (Figure 1). Merzenich et al. [26] surgically sutured the fingers of monkeys and then compared the pre- and post-surgical somatotopies of Brodmann area (BA) 3b, which corresponds to the sensorimotor area (SMA). Microelectrodes were used to record the responses in $\mathrm{BA} 3 \mathrm{~b}$ to finger stimuli. The third and fourth fingers were then surgically sutured, and the responses were recorded again a month later. Thus, the boundary between the third and fourth fingers became unclear. In addition, the results of a study that was conducted in human beings suggested that the plasticity of brain cells depends on sensory input. The results of a magnetoencephalography study that compared the somatotopies of the 


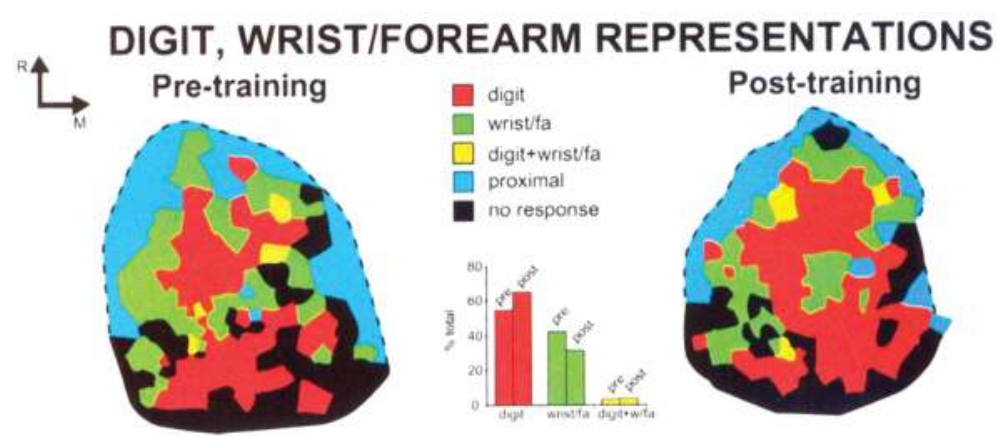

Figure 1. Representation of the distal forelimb in cortical area 4 derived from pre- and post-training mapping procedures [8].

first and fifth fingers of string players to normal controls showed that a broader cerebral cortical area was activated for string players compared to the controls [6].

These findings suggest that the size of the intracerebral somatotopic representation, which is vital to $\mathrm{ME}$, is determined by the degree of use of the region. If you try to induce plasticity in specific parts of the bodies of stroke patients, as mentioned above, the induction of neural plasticity in a pathway that allows highly efficient information processing by repeating movements in a pattern like the normal pattern should be possible, provided the patient has retained their motor functions to a certain degree. However, if a patient has the functional level of almost not able to perform movement or is only able to perform the movement in an abnormal pattern, the stimulation of the plasticity for the formation of a neural network that is required to be able to regain normal motor function may not be possible. Ward et al. [27] chronologically examined the relationships between motor function recovery scores and task-related brain activities for approximately 12 months after the onset of stroke with functional magnetic resonance imaging. They found a negative correlation between motor function recovery scores and a decline in the hyperactivity of brain areas in the damaged and undamaged hemispheres (M1, premotor cortex; PMC, supplementary motor cortex; SMC, cerebellum). These findings suggest that a better recovery of motor function is associated with better connectivity between the functional systems of multiple brain regions and that a continuous and long-term approach is required to study the changes in the morphologies and networks of neurons. Thus, a qualitative and continuous approach [28] is required in studies of the recovery of the entire neural system (e.g. transcortical network, M1-PMC neural network [29]) in order to be able to perform movement rather than merely establishing quantitative interventions of movement. Thus, next, we will discuss the current understanding of what is required in interventions for stroke patients.

\section{Interventions for stroke}

The establishment of a series of tripartite relationships between actual physical movement, a sense of ownership or agency, and imaging of physical exercise is an important component of 
NBR, rather than only involving actual physical movement for patients with motor or sensory disturbances or patients who lost their sense of body presence. Recently, decreased grey matter has been shown in multiple motor areas, and it affects the prognosis of motor function recovery [30]. The similar results of a study of monkeys that developed brain infarcts in their M1 area showed that the fiber connections between M1 and the hand brain area of the ventral premotor cortex (PMv) were altered [31]. The observations were conducted prior to and 5 months after the induction of the infarct. Prior to the infarct, M1 was observed to be connected with the SMC, $\mathrm{PMv}$, the dorsal premotor cortex, primary somatosensory cortex, and secondary somatosensory cortex by fibers, and these connections with M1 ceased to exist 5 months later. In contrast, the PMv was connected with M1 and the frontal rostral area prior to the infarct, and these connections with M1 ceased to exist after 5 months. However, a new network was formed between PMv and the primary somatosensory cortex. The results of these studies demonstrate that a cortical somatotopic representation is not a simple schema of the projection of one point to another point but rather a pervasive pattern in a broader range of the cortex, and a network that is based on the functional connectivity between areas is dynamically reconfigured by experience in order to reorganize the representation. These findings not only confirm the hypothesis that $\mathrm{ME}$ involves a large number of cerebral areas, including the motor areas but also implies that a simple movement treatment is not sufficient for functional recovery and that an intervention of a brain function network, including the relevant brain areas, is required.

Sharma et al. [32] classified the intervention components of previous studies that positively affected motor function recovery after a brain infarct into three concepts of approach: (i) sensory feedback, (ii) discharge through the corticospinal tract to produce movement, and (iii) motor processes that precede movement. The first concept, sensory feedback, is an approach that uses feedback from visual and auditory perception as well as somatosensory information to promote the recovery of motor functions. Sensory feedback implies that an enhancement of the nervous activity in the M1 $4 \mathrm{p}$ area through somatosensory input is related to the recovery of motor functions. The human M1 consists of two distinct areas: 4a and 4p [33]. The 4a and 4p areas have different cellular structures and different receptor densities. 4a is located in the anterior (rostral) part of M1, and it is called old M1 because it is a phylogenetically old area. Output from the old M1 controls physical movement through the corticospinal tract and spinal interneurons. In contrast, $4 \mathrm{p}$ is located in the posterior (caudal) part of the primary motor cortex, and it is called new M1 because it is a relatively new motor cortex. New M1 contains motor cortical neuronal cells that will be directly connected with spinal motor neurons through synapses. These synaptic connections are not mediated by spinal interneurons, and they are involved in the formation of highly skilled and complex movements [34]. Besides the structural difference, a functional difference in somatosensory afferent information processing has been found between $4 \mathrm{a}$ and $4 \mathrm{p}$. A study of the differences in the neural activities of $4 \mathrm{a}$ and $4 \mathrm{~b}$ in the motor area of monkeys in response to the inputs of different sensory modalities [35] showed that $4 \mathrm{a}$ is rich in cells that respond to the proprioceptive sensory inputs of muscles and joints, and $4 \mathrm{p}$ is rich in cells that respond to cutaneous sensory input. These findings suggested that paralyzed limbs need to be given cutaneous sensory input in order to enhance the excitability of $4 \mathrm{p}$. In addition, $4 \mathrm{p}$ is affected by active attention. In order to enhance the neural activity of $4 \mathrm{p}$, it is important to attract the active attention of the target subject toward the PT, in addition to simply providing him or her 
sensory stimuli. The second concept of discharge through the corticospinal tract to produce movement is an approach that requires the utilization of the corticospinal tract through the positive and intense production of movements. Task-oriented training can be produce by a typical intervention. This training, which consists of several components, is considered an intervention with a high evidence level [36]. The components include the provision of sufficient amounts of movement stress, gradual adjustment of the task difficulty, and feedback utilization. Sufficient amounts of repetitive training and training time need to be provided while rigorously setting up purposive tasks rather than having them repeat simple movements without purpose. Moreover, the most important point is that the intervention should not be a simple increase in the repetition of a movement but rather a step-by-step adjustment in task difficulty [37]. Therapists must be able to appropriately set and adjust the task difficulty according to the functional state of the patients.

Interventions that are based on concept (i) and (ii) stated thus far must involve proper physical movements so that patients do not learn wrong movements while processing actual sensorimotor information. The performance of skilled movement learning tasks is the most important component in PT. The learning of motor skills has been revealed to occur not only during movement practice (online learning) but also during sessions in which a subject is not practicing movement (offline learning) [38]. It is important to consider how offline learning should be implemented in an intervention. The third concept of motor processes that precede movement sheds light on this. Motor processes that precede movement represent the process of simulating a movement. Several mental practices have been developed to explicitly activate this process. However, while some stroke patients are in a brain functional state in which they are able to perform mental practice while actively imagining movements (top-down process), other stoke patients are not. If a top-down intervention is difficult, it is necessary to begin by intervening based on sensory information input (bottom-up process), reconstructing the body schema toward ME, and enhancing the intracerebral body representation capability. In addition, an important strategy of NBR is to ultimately maintain the coherency of the information that is processed and constructed in the brain by the top-down and bottom-up processes.

\section{Therapy based on voluntary top-down processing}

MI $[39,40]$ is a top-down process involving the active imagining of various states in the mind, rather than a bottom-up process of generating motion-related perceptions based on sensory input. Although the imagery in this context represents a mental image, MI is unlike visual imagery which is induced by the input of sensory information to the body in that MI is pragmatic, whereas visual imagery is semantic [41]. The intracerebral information processing in MI involves not only the formulation of a movement plan but also the cognitive manipulation of what has been imagined [13] as well as elements of self-image (first-person image) and images of others (third-person image) [42]. In addition, because imagery occurs by activating cognitive processing that utilizes working memory [43, 44], MI can also be defined as the mental motor representation that is reproduced by working memory.

Hétu et al. [45] reported that MI is produced in several brain areas, including the cerebellum, inferior/superior parietal lobule, precentral gyrus, inferior frontal gyrus, middle frontal 
gyrus, and SMC (Figure 2). Of these areas, the SMC is involved during the imagining of both motor images of the upper limbs and lower limbs, and the areas of the SMC that are active during MI and ME partially overlap [46]. The SMC is thought to be involved in the formation and manipulation of internal representations of movement in the brain when there are no extraneous stimuli or clues [47]. Because MI only lacks the actual movements and sensory feedback that occurs during ME, it can be considered functionally equivalent to ME except for these processes. Jeannerod et al. [48] stated that MI and ME are not distinct brain processes and that they differ only in the brain process concerning movement. Moreover, a study that examined and compared the brain activities of healthy subjects and stroke patients while they were performing ME, MI, passive movement, and movement observation [49] revealed that the MI brain activity of patients resembled the ME brain activity of healthy subjects and that MI is suited for sensorimotor system activation. In addition, the corticospinal tract is excited through its connection with MI. During transcranial magnetic

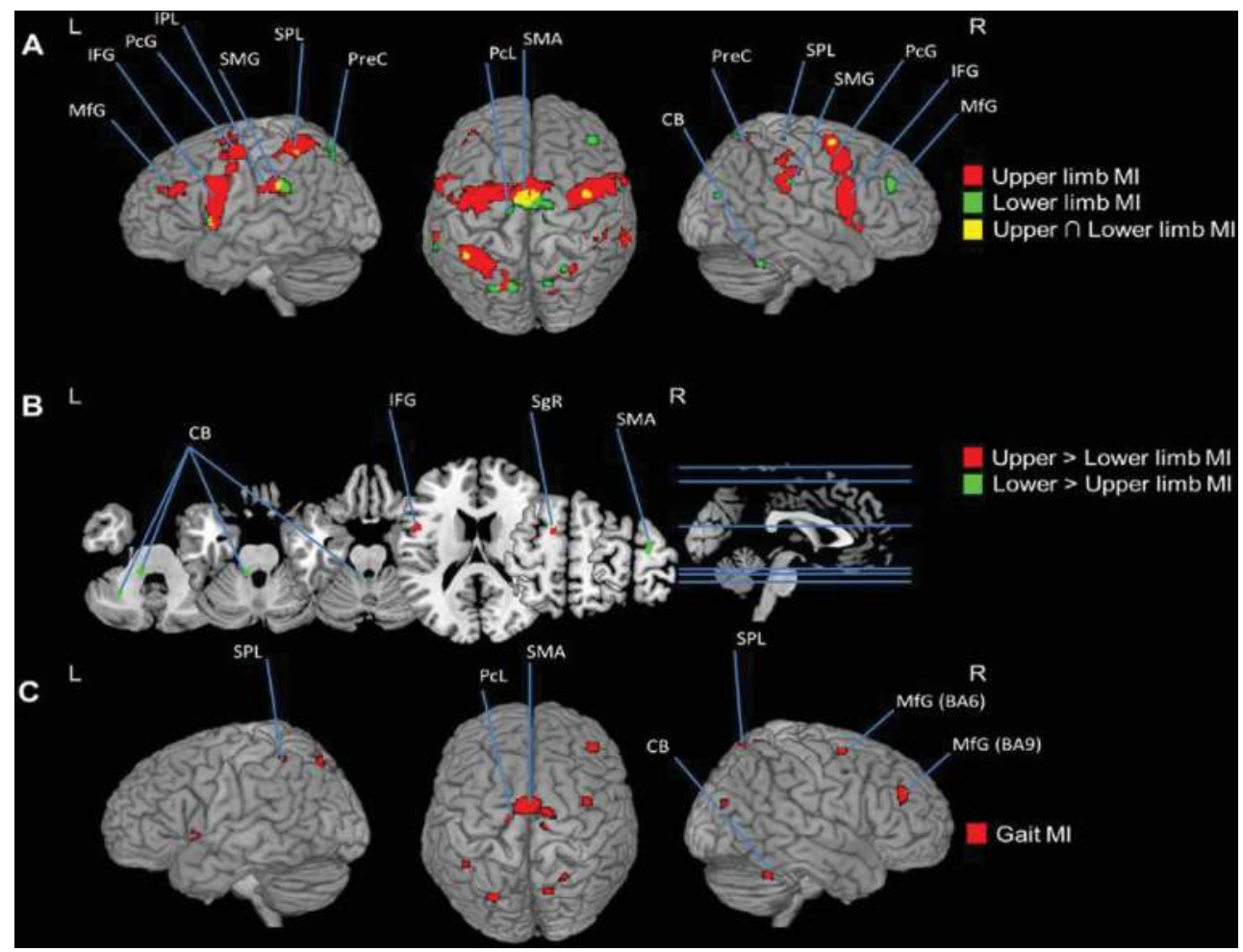

Figure 2. Regions consistently activated during motor imagery of the upper and lower limbs [45]. A: Maps of consistent activations while subjects imagined movements of the upper (red) or lower (green) limbs. Regions consistently activated by both types of movements are shown in yellow. B: Results of the subtraction analysis: regions with more consistent activity during motor imagery of upper limbs are shown in red and of lower limbs in green. C: Regions consistently activated while imagining gait movements. CB: cerebellum; IPL/SPL: inferior/superior parietal lobule; PcG: precentral gyrus; IFG: inferior frontal gyrus; MfG: middle frontal gyrus; SMA: supplementary motor area; SMG: supramarginal gyrus; PreC: precuneus; PcL: paracentral lobule; SgR: subgyral region. 
stimulation, the threshold of motor-evoked potentials (MEP) and intracortical facilitation decrease, and a latent time reduction and amplitude increment are observed. In the neurotransmission test, a spinal H-reflex amplitude increment and increase in the F-wave frequency rate have been reported [50]. Electroencephalography (EEG) shows that the formation of MI, like ME, reduces the $\alpha$-wave (Mu-rhythm) amplitude in the SMA (Refer to Section Interventions based on extraneous bottom-up processes), which is called event-related desynchronization (ERD). The degree of change rate in ERD that is associated with MI correlates with the motor-evoked potential increment, intracortical inhibition reduction, and F-wave frequency rate, and ERD is thought to reflect the excitability of the corticospinal tract [51].

However, activity in M1, which is the final area of motor output, during MI is debatable (Figure 3) [45]. As reported by studies by Sharma et al. [52] and Ehrsson et al. [53], some studies report M1 neural activity during MI [54, 55], whereas others report that no activity was observed, and whether it is active or not varies according to each subject. MI is also thought to reflect a suppression process so that the actual movement is not performed The SMC has been reported to inhibit M1 activation during MI [48, 56], which then causes ME to cease [57]. Thus, the different reports on activation can be explained by the finding that the information that is processed during MI is supposed to be inhibited so that ME does not occur, which has sometimes been conveyed to M1 Through the contamination of an image by muscle activity [50].

Two components have been revealed to exist in the information processing system for generating MI in the brain: one is the simulation of motor parameters, which generates a concrete motor execution plan, such as which muscles should be moved and how in movement execution, and the other is the simulation of motor perception, which predictively generates a motor execution plan that is based on expected motor perceptions during the motor preparation phase. Because network activities in a number of areas, such as the frontal lobe, basal ganglia, and cerebellum, are involved in this system [58], it is considered necessary during PT to intervene and direct the patients by asking them to imagine the direction of a movement and the muscles that must be moved to perform the movement or imagine the motor perception that accompanies movement and the perception that forms between themselves and an object in order to leverage all these areas. Furthermore, the observation that MI changes intracerebral neural activities and the excitability of the corticospinal tract suggests the possibility that $\mathrm{MI}$ is a useful intervention method in PT. An intervention that has recently used ERD through MI is the brain machine interface (BMI).

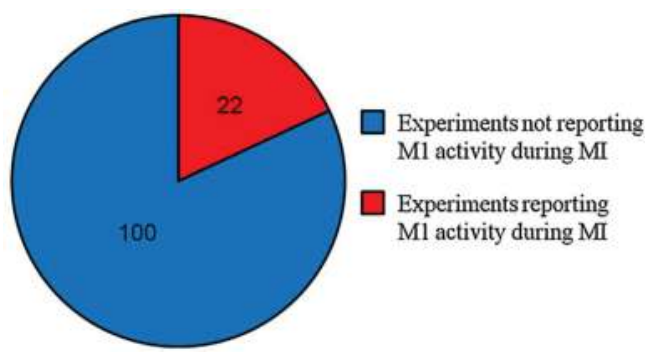

Figure 3. Proportion of neuroimaging experiments on motor imagery where activity in the primary motor cortex (M1) was found [45]. 
Among the rehabilitation therapies that use BMI, there is a functional compensation BMI that aims to compensate for the loss of function, and a functional recovery BMI that aims to recover functions. In particular, a strategy of functional recovery BMI that has been proposed is the use of robots to support near-normal movement and generate correct sensory inputs to induce plasticity in brain neurons [59]. A study by Ramos-Murguialday et al. [60] that examined the effects of functional recovery BMI and that utilized MI reported the results of a randomized comparison test that examined the effects of BMI training on 32 cases of chronic stroke patients who had difficulty extending the fingers on their paralyzed hand by dividing them into the BMI-trained group and the control group. In the BMI training, ERD during MI was analyzed, and the brain moved the upper limb robot and the finger orthosis in accordance with the changes (Figure 4). The control group moved their hands randomly. Because of an intervention that was performed for an average of 17.8 days, the paralyzed side of the upper limb functions improved significantly for the BMI-trained group compared to the control group. Furthermore, the improvements were reported to be related to an electromyographic amplitude value during voluntary muscle contraction as well as the degree of activity shift from the undamaged hemisphere to the damaged hemisphere on the functional MRI.

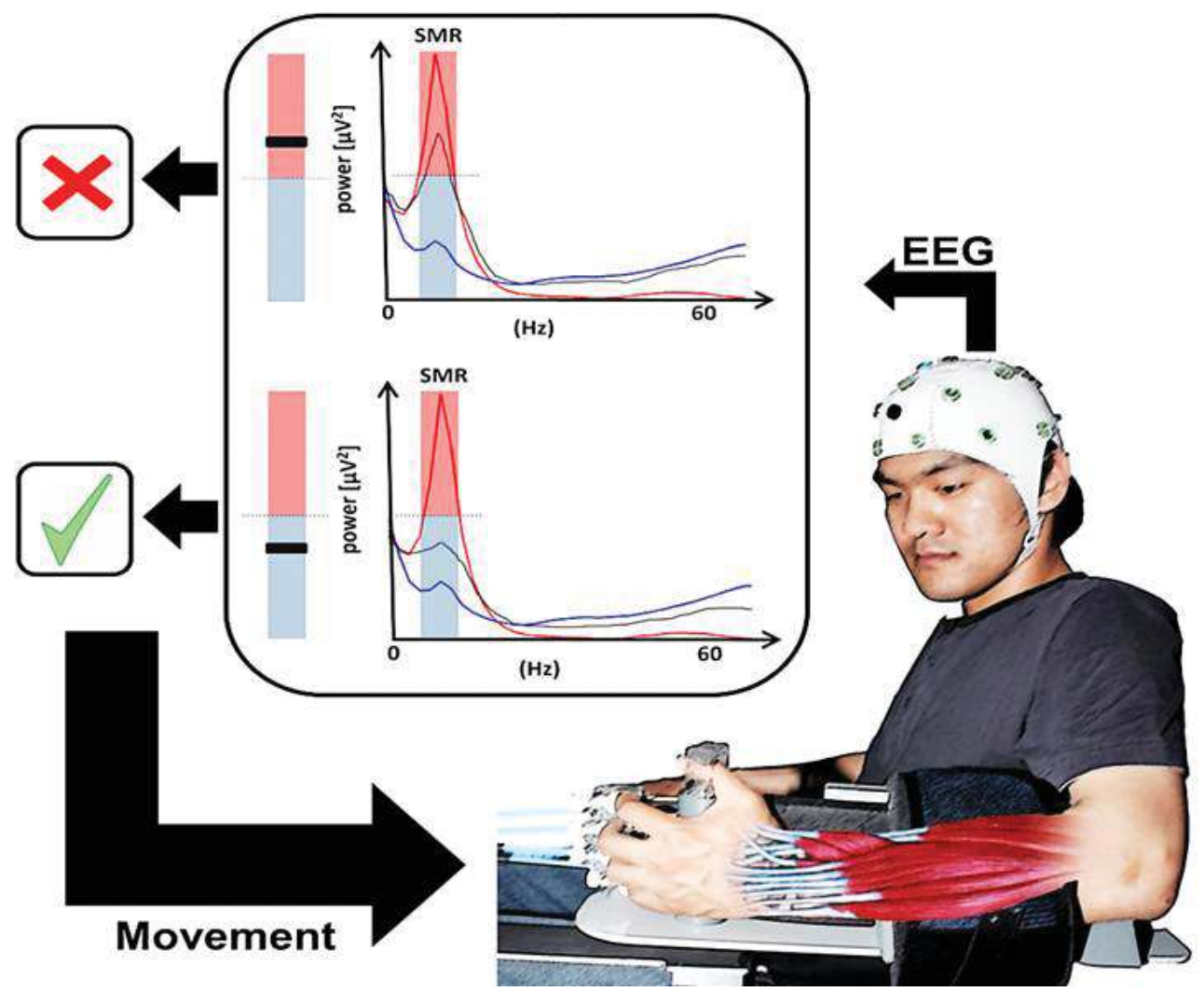

Figure 4. Brain-machine-interface in stroke [60]. 
With the recent development and advances in analysis and intervention tools, mental practice that utilizes MI is currently being established. However, because MI has problems that are related to vividness, controllability, immersion, and habitude, large individual differences in image recollection capabilities are suspected. The bottom-up-processed image that is based on visual information proceeds according to two distinct pathways: "the what pathway" (ventral stream), which processes shape and color and "the where pathway" (dorsal stream), which processes spatial locations and movements [61]. The processing occurs in a phased and hierarchical manner with the former processing object imagery and the latter processing spatial imagery. This enables us to understand image capability based on image capability assessments, such as the Object-Spatial Imagery Questionnaire [62]. However, a large number of studies have revealed that $\mathrm{MI}$ is processed in a top-down manner. An interesting study that shows this functional difference by Macrae and Troll et al. [63] reported that patients who could not imagine home and the way to go home nevertheless made no mistakes going home and exhibited no mistaken behavior at home. Because the recollection capability of MI involves memory that is related to one's own movements and movement experiences, it is possible that the body representation capability within the brain has largely declined in stroke patients who have suffered disturbances of their sensorimotor functions for an extended period of time since onset, which makes it impossible to recollect MI. For these cases, the motor sensory information input needs to occur in a bottom-up manner and the sense of ownership needs to reorganize itself.

\section{Interventions based on extraneous bottom-up processes}

When the brain controls bodily movements, the proprioceptors in muscles largely contribute to their realization. The great contribution of proprioception to motor control is revealed by the observation that patients with proprioception disturbances cannot move their fingers very well [64]. In addition, this perception is deeply involved in the intracerebral body representation. Two concepts of body image [65] and body schema [66] have been proposed for body representation. Of the two, the body schema, which is a model of one's own posture that is updated every second according to the sensory information input, is an intracerebral body representation before it is brought to consciousness. If the treatment goal is to regain motor control in PT for stroke patients, it is difficult to regain smooth and predictive motor control only by interventions in the manner of conscious control or control based on visual information. Because many limb movements are executed unconsciously, we considered them an important element in NBR for regenerating a body schema that is based on input from proprioception [65]. Based on these ideas, we present the details of a study [20] that utilized kinaesthetic illusions that were induced in the brain by tendon vibration to examine how they affected the neural functions of stroke patients.

\subsection{Experiment}

There have been many reports on the efficacy of treatment using illusory kinaesthesia for NBR in patients with a cerebrovascular accident (CVA). Illusory kinaesthesia is defined as an illusion in which the subject feels the movement of their limbs by extrinsic and/or intrinsic stimuli without actual voluntary movement [67]. Illusory kinaesthesia evoked by sensorimotor information in the brain activates brain nerve activity, and the neural activity can be considered as the neural basis of motor control and can be applied for motor learning [68]. When illusory kinaesthesia is used as a 
treatment, it is required to predict sensory information that would be inputted prior to the illusory movement and to simulate previous sensory movement experiences as the motor image [69]. Thus, it is important to assess whether this brain neural process observed in healthy adults can also occur in patients with a CVA to understand the efficacy of the approach as an intervention. In this experiment, to understand the functional brain status from the time when the motor imaging is started prior to the illusory kinaesthesia, we evaluated how illusory kinaesthesia influences brain neural activity in patients with CVAs using electroencephalography (EEG).

\subsubsection{Methods}

\subsubsection{Subjects}

Subjects were 12 healthy adults who did not have any orthopedic or neurological diseases and who were without motor and sensory disorders (healthy control group) and 13 patients with CVAs (CVA group). In the CVA group, subjects did not have any cognitive dysfunction and superficial and deep sensory tract disorders.

\subsubsection{Experimental conditions and neurophysiological outcomes}

For vibratory stimulation, vibration was applied to the tendon of the distal part of the right biceps, and the target movement of illusory kinaesthesia was elbow extension. As the neurophysiological outcome of the influence of illusory kinaesthesia on brain neural activity, we used mu-rhythm ( $\mu$-wave), which is one of the frequency components of the brain wave and is the same frequency band as the $\alpha$ wave band. The $\mu$-wave decreases not only during actual movement but also during motor imaging [70,71]; thus, it can be an outcome of brain neural activity, including the generation process, during illusory kinaesthesia around the sensorimotor area. For vibratory stimulation, a handheld massager (THRIVE MD-01, Thrive co., Ltd) was used. Since the vibratory stimulation was applied on the skin, it could stimulate not only the muscle spindles but also the superficial sensory receptors. The $\mu$-wave, which changes depending on the sensorimotor information, can also be affected by sensory information from cutaneous stimulation [72]. To control these effects, we subtracted the EEG data obtained during the contact of the vibratory device on the right biceps tendon without vibratory stimulation from the EEG data when applying vibratory stimulation to evoke illusory kinaesthesia to obtain data for analysis. The frequency of the vibratory stimulation was set at $91.7 \mathrm{~Hz}[67,73,74]$.

\subsubsection{EEG analysis}

We used Neurofax (Nihon Koden) to record EEGs. The measurement site was based on the international 10-20 system, and EEGs were obtained from 18 electrodes with bilateral earlobes as reference electrodes. The recording time for each trial was 60 seconds [73].

In the EEG data that were controlled by sensory information in both the healthy control and CVA groups, to investigate brain activity in the $\mu$-wave band area, we first divided the waves into epochs of 2 seconds (30 epochs in total) each. We analyzed the cumulative mean of the data using the three-dimensional filtering of brain neural activity, that is, standardized low resolution brain electromagnetic tomography (sLORETA) [75, 76], to identify brain neural activity in the $\mu$-wave band area. For obtaining the results of the analysis, the brain neural 
activity area was calculated as the current density value simulated with 6234 voxels (MNI coordinates [77]) in the brain, and this was identified as the Brodmann area (BA). For comparison of the vibratory stimulation condition-specific differences in brain neural activity areas between the groups, we used sLORETA-based SnPM analysis [78].

\subsubsection{Results}

The brain neural activity in the $\mu$-wave ( $\alpha$ wave when it appears in the posterior lobe [79]) band area due to vibratory stimulation was significantly greater in the posterior lobe compared to the cerebral cortex area in both groups, while there was no significant neural activity in the sensorimotor area (Figure $\mathbf{5} \mathbf{a}$ and $\mathbf{b}$ ). For group differences, the healthy control group
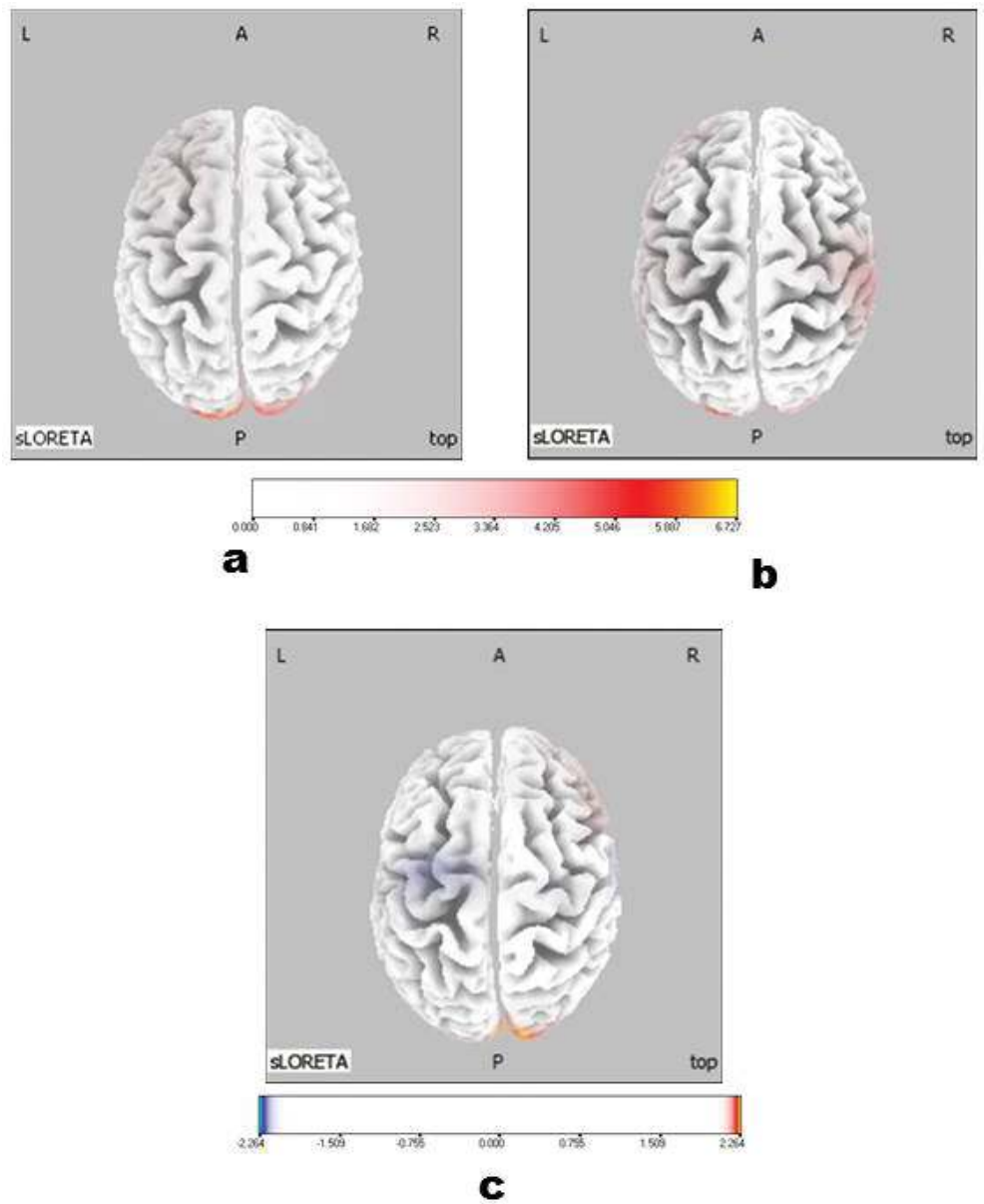

Figure 5. Brain neural activity in the $\mu$-wave band area due to vibratory stimulation. (a) Brain neural activity in the $\mu$ wave band area of the healthy control group, (b) brain neural activity in the $\mu$-wave band area of the CVA group, (c) comparison of brain neural activity in the $\mu$-wave band area between the healthy control and CVA groups. The neural activity in the SMC was significantly greater in the CVA group compared to the healthy control group. The color scale shown in the bottom of the image indicates the $t$-value $(t=2.264)$. 
had a significantly greater increase in neural activity in the posterior lobe compared to the CVA group, while the CVA group had a significantly greater increase in the SMC (Figure 5c).

\subsubsection{Discussion}

This study assessed the influence of illusory kinaesthesia evoked by vibratory stimulation on brain neural activity in patients with CVAs, using the EEG $\mu$-wave, which is a neurophysiological outcome.

As for the brain neural activity in the $\mu$-wave band area due to vibratory stimulation, we did not find significant expression of the $\mu$-wave in the sensorimotor area in both groups. This may be due to the increase in sensorimotor information in the bilateral sensorimotor-related area [80] causing event-related desynchronisation in neural activity, which decreased the $\mu$-wave [70, 71]. Furthermore, the neural activity in the M1 area, which is the main sensorimotor area, is not only involved in the generation of functional connectivity with BA3b, which deals with sensory information processing from the muscle spindles, but is also related to the cause of illusory kinaesthesia itself [81]. Thus, it is believed that the amount of perception of illusory kinaesthesia is greater when excitability of the motor cortex is greater [68]. Therefore, illusory kinaesthesia was evoked also in the CVA group by enhancing neural activity in the sensorimotor area using vibratory stimulation.

Furthermore, for a significant increase in the SMC in the CVA group compared to the healthy control group, the SMC stores evoked motor images of not only the constant sensorimotor feedback information during movement execution, but also the sensorimotor information during passive movement input, such as illusory kinaesthesia [69]. This was deeply related to spatial cognitive processes [82] in brain representations [83, 84]. Therefore, it was presumed that, in patients with CVAs who had movement deficits, there were some functional differences in the simulation process of the motor image during generation of illusory kinaesthesia compared to healthy adults. Furthermore, information processing to generate the illusion and completeness of the generated motor image may be different.

The results of our study showed that there might be functional differences in the generation mechanism of illusory kinaesthesia between healthy adults and adults with CVAs, while illusory kinaesthesia can also be evoked in patients, which increases the neural activity in the sensorimotor area and SMC.

\section{Toward a therapy that aims for unification}

The utilization of kinaesthetic illusions allows for the induction of realistic motor perceptions, which constitutes the neural basis of ME, while activating the sensorimotor area. This therapy can be used to help patients be aware of physical movements that they perform as well recover a sense of ownership while triggering neural reorganization. To make kinaesthetic illusions more vivid and realistic, the combination of $\mathrm{ME}$ and $\mathrm{MI}$ is required with the intention of motion. Naito et al. [19] reported that kinaesthetic illusions and hand MI collectively utilized the motor areas of the SMA, dorsal premotor cortex, and cerebellum. Between the body schema of MI and the body schema of kinaesthetic illusion lies the common neural basis. If patients intentionally create an MI of quasi movement while experiencing a kinaesthetic 
illusion, the experience of the kinaesthetic illusion is enhanced, which allows them to experience a more realistic movement [19]. These findings revealed that there is an interference between kinaesthetic illusions and MI. In NBR, the focus is on eliciting voluntary and active movements from the patients themselves. kinaesthetic illusions, which reflect the processes of the sensorimotor area that converts signals from proprioception to motor commands, is itself a neural mechanism that is directly connected with motor control. This will be a useful strategy for PT for patients with impaired motor functions to create an intention of movement through the MI and elicit the ME while evoking kinaesthetic illusions.

\section{Conclusion}

This paper presented several theories of the reorganization of brain functions in stroke patients that were based on neuroscientific evidence and an outline of such attempts. A therapist's accurate understanding of the patient's state results in increased options for treatment strategies. In addition, therapists have greater options for patients to have the possibility of an autonomous social life. However, we must understand the limitations and individual differences in functional recovery $[85,86]$. Thus, the application of the same therapy may not cause the same level of recovery. Furthermore, the induction of network reinforcement and fixation by the simple input of stimuli into neurons is difficult because mental acts by the patient are vital. PT becomes most effective when adequate interventions that induce neural network reorganization and positive states of mind are combined.

\section{Acknowledgements}

This study was part of a research program funded by the JSPS (research grant 15K01439). The funding source had no role in study design/concept, data collection/analysis/interpretation, and manuscript preparation/submission.

\section{Author details}

Takayuki Kodama* and Hideki Nakano

*Address all correspondence to: kodama-t@tachibana-u.ac.jp

Department of Physical Therapy, Faculty of Health Sciences, Kyoto Tachibana University, Kyoto, Japan

\section{References}

[1] Cajal S, Ramon Y: Degeneration \& Regeneration of the Nervous System. Trans. and edited by RM May ed. London: Oxford University Press; 1928. 
[2] Merzenich MM, Nelson RJ, Stryker MP, Cynader MS, Schoppmann A, Zook JM: Somatosensory cortical map changes following digit amputation in adult monkeys. J Comp Neurol. 1984;224(4):591-605.

[3] Donoghue JP, Suner S, Sanes JN: Dynamic organization of primary motor cortex output to target muscles in adult rats. II. Rapid reorganization following motor nerve lesions. Exp Brain Res. 1990;79(3):492-503.

[4] Cohen LG, Bandinelli S, Findley TW, Hallett M: Motor reorganization after upper limb amputation in man. A study with focal magnetic stimulation. Brain. 1991;114(Pt 1B): 615-627.

[5] Sterr A, Müller MM, Elbert T, Rockstroh B, Pantev C, Taub E: Changed perceptions in Braille readers. Nature. 1998;391(6663):134-135.

[6] Elbert T, Pantev C, Wienbruch C, Rockstroh B, Taub E: Increased cortical representation of the fingers of the left hand in string players. Science. 1995;270(5234):305-307.

[7] Calautti C, Baron JC: Functional neuroimaging studies of motor recovery after stroke in adults: a review. Stroke. 2003;34(6):1553-1566.

[8] Nudo RJ, Milliken GW, Jenkins WM, Merzenich MM: Use-dependent alterations of movement representations in primary motor cortex of adult squirrel monkeys. J Neurosci. 1996;16(2):785-807.

[9] Hayward KS, Brauer SG: Dose of arm activity training during acute and subacute rehabilitation post stroke: a systematic review of the literature. Clin Rehabil. 2015;29(12):1234-1243.

[10] Döbrössy MD, Dunnett SB: The influence of environment and experience on neural grafts. Nat Rev Neurosci. 2001;2(12):871-879.

[11] Pollock A, Farmer SE, Brady MC, Langhorne P, Mead GE, Mehrholz J, van Wijck F: Interventions for improving upper limb function after stroke. Cochrane Database Syst Rev. 2014;11:CD010820.

[12] Guillot A, Di Rienzo F, Collet C: The neurofunctional architecture of motor imagery, in Advanced Brain Neuroimaging Topics in Health and Disease-Methods and Applications, Papageorgiou TD, Christopoulos GI, Smirnakis SM, editors. (Rijeka: InTech;) 2014: 433-456.

[13] Decety J: The neurophysiological basis of motor imagery. Behav Brain Res. 1996;77 (1-2):45-52.

[14] Molina-Luna K, Hertler B, Buitrago MM, Luft AR: Motor learning transiently changes cortical somatotopy. Neuroimage. 2008;40(4):1748-1754.

[15] Jenkinson PM, Haggard P, Ferreira NC, Fotopoulou A: Body ownership and attention in the mirror: insights from somatoparaphrenia and the rubber hand illusion. Neuropsychologia. 2013;51(8):1453-1462.

[16] Garbarini F, Fossataro C, Berti A, Gindri P, Romano D, Pia L, della Gatta F, Maravita A, Neppi-Modona M: When your arm becomes mine: pathological embodiment of alien 
limbs using tools modulates own body representation. Neuropsychologia. 2015;70: 402-413.

[17] Lackner JR, Taublieb AB: Reciprocal interactions between the position sense representations of the two forearms. J Neurosci. 1983;3:2280-2285.

[18] Roll JP, Gilhodes JC: Proprioceptive sensory codes mediating movement trajectory perception: human hand vibration-induced drawing illusions. Can J Physiol Pharmacol. 1995;73(2):295-304.

[19] Naito E, Kochiyama T, Kitada R, Nakamura S, Matsumura M, Yonekura Y, Sadato N: Internally simulated movement sensations during motor imagery activate cortical motor areas and the cerebellum. J Neurosci. 2002;22(9):3683-3691.

[20] Kodama T, Nakano H, Ohsugi H, Murata S: Effects of vibratory stimulation-induced kinesthetic illusions on the neural activities of patients with stroke. J Phys Ther Sci. 2016;28(2):419-425.

[21] Eriksson PS, Perfilieva1 E, Björk-Eriksson T, Alborn AM, Nordborg C, Peterson DA, Gage FH: Neurogenesis in the adult human hippocampus. Nat Med. 1998;4:1313-1317.

[22] Murphy TH, Corbett D: Plasticity during stroke recovery: from synapse to behaviour. Nat Rev Neurosci. 2009;10(12):861-872.

[23] Toni N, Buchs PA, Nikonenko I, Bron CR, Muller D: LTP promotes formation of multiple spine synapses between a single axon terminal and a dendrite. Nature. 1999;402(6760):421-425.

[24] Hebb DO: The organization of behavior: a neuropsychological theory. New York: Wiley \& Sons; 1949.

[25] Turrigiano GG, Leslie KR, Desai NS, Rutherford LC, Nelson SB: Activity-dependent scaling of quantal amplitude in neocortical neurons. Nature. 1998;391:892-896.

[26] Merzenich MM, Kaas JH, Wall J, Nelson RJ, Sur M, Felleman D: Topographic reorganization of somatosensory cortical areas $3 \mathrm{~b}$ and 1 in adult monkeys following restricted deafferentation. Neuroscience. 1983;8(1):33-55.

[27] Ward NS, Brown MM, Thompson AJ, Frackowiak RS: Neural correlates of motor recovery after stroke: a longitudinal fMRI study. Brain. 2003;126:2476-2496.

[28] Grefkes C, Ward NS: Cortical reorganization after stroke: how much and how functional? Neuroscientist. 2014;20(1):56-70.

[29] Kantak SS, Stinear JW, Buch ER, Cohen LG: Rewiring the brain: potential role of the premotor cortex in motor control, learning, and recovery of function following brain injury. Neurorehabil Neural Repair. 2012;26(3):282-292.

[30] Gauthier LV, Taub E, Mark VW, Barghi A, Uswatte G: Atrophy of spared gray matter tissue predicts poorer motor recovery and rehabilitation response in chronic stroke. Stroke. 2012;43(2):453-457. 
[31] Nudo RJ: Postinfarct cortical plasticity and behavioral recovery. Stroke. 2007;38(Suppl 2):840-845.

[32] Sharma N, Cohen LG: Recovery of motor function after stroke. Dev Psychobiol. 2012;54 (3):254-262.

[33] Geyer S, Ledberg A, Schleicher A, Kinomura S, Schormann T, Bürgel U, Klingberg T, Larsson J, Zilles K, Roland PE: Two different areas within the primary motor cortex of man. Nature. 1996;382:805-807.

[34] Rathelot JA, Strick PL: Subdivisions of primary motor cortex based on cortico-motoneuronal cells. Proc Natl Acad Sci USA. 2009;106(3):918-923.

[35] Strick PL, Preston JB: Sorting of somatosensory afferent information in primate motor cortex. Brain Res. 1978;156:364-368.

[36] Timmermans AA, Spooren AI, Kingma H, Seelen HA: Influence of task-oriented training content on skilled arm-hand performance in stroke: a systematic review. Neurorehabil Neural Repair. 2010;24(9):858-870.

[37] Plautz EJ, Milliken GW, Nudo RJ: Effects of repetitive motor training on movement representations in adult squirrel monkeys: role of use versus learning. Neurobiol Learn Mem. 2000;74(1):27-55.

[38] Dayan E, Cohen LG: Neuroplasticity subserving motor skill learning. Neuron. 2011;72 (3):443-454.

[39] Jeannerod M: The representing brain: neural correlates of motor intention and imagery. Behav Brain Sci. 1994;17(2):187-202.

[40] Bakker M, de Lange FP, Stevens JA, Toni I, Bloem BR: Motor imagery of gait: a quantitative approach. Exp Brain Res. 2007;179(3):497-504.

[41] McAvinue LP, Robertson IH: Relationship between visual and motor imagery. Percept Mot Skills. 2007;104(3 Pt 1):823-843.

[42] Hanakawa T, Immisch I, Toma K, Dimyan MA, Van Gelderen P, Hallett M: Functional properties of brain areas associated with motor execution and imagery. J Neurophysiol. 2003;89:989-1002.

[43] Erro R, Hirschbichler ST, Ricciardi L, Ryterska A, Antelmi E, Ganos C, Cordivari C, Tinazzi M, Edwards MJ, Bhatia KP: Mental rotation and working memory in musicians' dystonia. Brain Cogn. 2016;109:124-129.

[44] Farah MJ: The neural basis of mental imagery. Trends Neurosci. 1989;12(10):395-399.

[45] Hétu S, Grégoire M, Saimpont A, Coll MP, Eugène F, Michon PE, Jackson PL: The neural network of motor imagery: an ALE meta-analysis. Neurosci Biobehav Rev. 2013;37 (5):930-949. 
[46] Deiber MP, Ibañez V, Sadato N, Hallett M: Cerebral structures participating in motor preparation in humans: a positron emission tomography study. J Neurophysiol. 1996;75 (1):233-247.

[47] Stephan KM, Fink GR, Passingham RE, Silbersweig D, Ceballos-Baumann AO, Frith CD, Frackowiak RS: Functional anatomy of the mental representation of upper extremity movements in healthy subjects. J Neurophysiol. 1995;73(1):373-386.

[48] Jeannerod M: Neural simulation of action: a unifying mechanism for motor cognition. Neuroimage. 2001;14(1 Pt 2):S103-S109.

[49] Szameitat AJ, Shen S, Conforto A, Sterr A: Cortical activation during executed, imagined, observed, and passive wrist movements in healthy volunteers and stroke patients. Neuroimage. 2012;62(1):266-280.

[50] Di Rienzo F, Collet C, Hoyek N, Guillot A: Impact of neurologic deficits on motor imagery: a systematic review of clinical evaluations. Neuropsychol Rev. 2014;24(2):116-147.

[51] Takemi M, Masakado Y, Liu M, Ushiba J: Event-related desynchronization reflects downregulation of intracortical inhibition in human primary motor cortex. J Neurophysiol. 2013;110(5):1158-1166.

[52] Sharma N, Jones PS, Carpenter TA, Baron JC: Mapping the involvement of BA 4a and 4p during motor imagery. Neuroimage. 2008;41(1):92-99.

[53] Ehrsson HH, Geyer S, Naito E: Imagery of voluntary movement of fingers, toes, and tongue activates corresponding body-part-specific motor representations. J Neurophysiol. 2003;90(5):3304-3316.

[54] Guillot A, Collet C, Nguyen VA, Malouin F, Richards C, Doyon J: Functional neuroanatomical networks associated with expertise in motor imagery. Neuroimage. 2008;41 (4):1471-1483.

[55] Solodkin A, Hlustik P, Chen EE, Small SL: Fine modulation in network activation during motor execution and motor imagery. Cereb Cortex. 2004;14(11):1246-1255.

[56] Kasess CH, Windischberger C, Cunnington R, Lanzenberger R, Pezawas L, Moser E: The suppressive influence of SMA on M1 in motor imagery revealed by fMRI and dynamic causal modeling. Neuroimage. 2008;40(2):828-837.

[57] Guillot A, Di Rienzo F, Macintyre T, Moran A, Collet C: Imagining is not doing but involves specific motor commands: a review of experimental data related to motor inhibition. Front Hum Neurosci. 2012;6:247.

[58] Lorey B, Pilgramm S, Bischoff M, Stark R, Vaitl D, Kindermann S, Munzert J, Zentgraf K: Activation of the parieto-premotor network is associated with vivid motor imagery-a parametric fMRI study. PLoS One. 2011;6(5):e20368.

[59] Daly JJ, Wolpaw JR: Brain-computer interfaces in neurological rehabilitation. Lancet Neurol. 2008;7(11):1032-1043. 
[60] Ramos-Murguialday A, Broetz D, Rea M, Läer L, Yilmaz O, Brasil FL, Liberati G, Curado MR, Garcia-Cossio E, Vyziotis A, Cho W, Agostini M, Soares E, Soekadar S, Caria A, Cohen LG, Birbaumer N: Brain-machine interface in chronic stroke rehabilitation: a controlled study. Ann Neurol. 2013;74(1):100-108.

[61] Goodale MA, Westwood DA, Milner AD: Two distinct modes of control for object-directed action. Prog Brain Res. 2004;144:131-144.

[62] Blajenkova O, Kozhevnikov M, Motes MA: Object-spatial imagery: a new self-report imagery questionnaire. Appl Cogn Psychol. 2006;20(2);239-263.

[63] Macrae D, Troll E: The defect of function in visual agnosia. Brain. 1956;79:94-110.

[64] Ghez C, Gordon J, Ghilardi MF: Impairments of reaching movements in patients without proprioception. II. Effects of visual information on accuracy. J Neurophysiol. 1995;73 (1):361-372.

[65] Naito E, Morita T, Amemiya K: Body representations in the human brain revealed by kinesthetic illusions and their essential contributions to motor control and corporeal awareness. Neurosci Res. 2016;104:16-30.

[66] Head H, Holmes G: Sensory disturbances from cerebral lesions. Brain. 1911;34:102-254.

[67] Goodwin GM, McCloskey DI, Matthews PBC: The contribution of muscle afferents to kinaesthesia shown by vibration induced illusions of movement and by the effects of paralysing joint afferents. Brain. 1972;95:705-748.

[68] Naito E, Roland PE, Ehrsson HH: I feel my hand moving: a new role of the primary motor cortex in somatic perception of limb movement. Neuron. 2002;36:979-988.

[69] Harada T, Saito DN, Kashikura K, Sato T, Yonekura Y, Honda M, Sadato N: Asymmetrical neural substrates of tactile discrimination in humans: a functional magnetic resonance imaging study. J Neurosci. 2004;24:7524-7530.

[70] Pfurtscheller G, Neuper C: Motor imagery activates primary sensorimotor area in humans. Neurosci Lett. 1997;239:65-68.

[71] Muthukumaraswamy SD, Johnson BW, McNair NA: Mu rhythm modulation during observation of an object-directed grasp. Brain Res Cogn Brain Res. 2004;19:195-201.

[72] Naito E, Nakashima T, Kito T, Aramaki Y, Okada T, Sadato N: Human limb-specific and non-limb-specific brain representations during kinesthetic illusory movements of the upper and lower extremities. Eur Neurosci. 2007;25:3476-3487.

[73] Naito E, Ehrsson HH, Geyer S, Zilles K, Roland PE: Illusory arm movements activate cortical motor areas: a positron emission tomography study. J Neurosci. 1999;19: 6134-6144.

[74] Burke D, Hagbarth KE, Löfstedt L, Wallin BG: The responses of human muscle spindle endings to vibration during isometric contraction. J Physiol. 1976;261:695-711. 
[75] Pascual-Marqui RD, Michel CM, Lehmann D: Low resolution brain electromagnetic tomography: a new method for localizing electrical activity in the brain. Int J Psychophysiol. 1994;18:49-65.

[76] Pascual-Marqui RD: Standardized low-resolution brain electromagnetic tomography (sLORETA): technical details. Methods Find Exp Clin Pharmacol. 2002;24:5-12.

[77] Collins DL, Holmes CJ, Peters TK, et al.: Automatic 3-D model-based neuroanatomical segmentation. Hum Brain Mapping. 1995;3:190-208.

[78] Pascual-Marqui RD: Instantaneous and lagged measurements of linear and nonlinear dependence between groups of multivariate time series: frequency decomposition. arXiv. 2007;0711:1455.

[79] Sadato N, Nakamura S, Oohashi T, et al.: Neural networks for generation and suppression of alpha rhythm: a PET study. Neuroreport. 1998;9:893-897.

[80] Tanji J, Okano K, Sato KC: Neuronal activity in cortical motor areas related to ipsilateral, contralateral, and bilateral digit movements of the monkey. J Neurophysiol. 1988;60:325342.

[81] Naito E: Sensing limb movements in the motor cortex: how humans sense limb movement. Neuroscientist. 2004;10:73-82.

[82] Boussaoud D: Attention versus intention in the primate premotor cortex. Neuroimage. 2001;14:40-45.

[83] Van de Winckel A, Sunaert S, Wenderoth N, et al.: Passive somatosensory discrimination tasks in healthy volunteers: differential networks involved in familiar versus unfamiliar shape and length discrimination. Neuroimage. 2005;26:441-453.

[84] Chung GH, Han YM, Jeong SH, et al.: Functional heterogeneity of the supplementary motor area. AJNR Am J Neuroradiol. 2005;26:1819-1823.

[85] Rowland LM, Shadmehr R, Kravitz D, Holcomb HH: Sequential neural changes during motor learning in schizophrenia. Psychiatry Res. 2008;163(1):1-12.

[86] Pezawas L, Verchinski BA, Mattay VS, Callicott JH, Kolachana BS, Straub RE, Egan MF, Meyer-Lindenberg A, Weinberger DR: The brain-derived neurotrophic factor val 66 met polymorphism and variation in human cortical morphology. J Neurosci. 2004;24:1009910102. 